\title{
Epidemiología y patogenia de la hipertensión arterial esencial, rol de angiotensina II, óxido nítrico y endotelina
}

Eduardo Fiedler-Velásquez, Charles Gourzong-Taylor

Servicio Medicina Interna Hospital Calderón Guardia

Abreviaturas: HTA, T hipertensión arterial; All, angiotensina II; NO, oxido nítrico; PAS, presión arterial sistólica; PAD, presión arterial diastólica; SRA, sistema renina angiotensina aldosterona; ECA, enzima convertidota de angiotensina; IAM, infarto agudo al miocardio.

\section{Correspondencia:}

Eduardo Fiedler-Velásquez

Hospital Calderón Guardia

Correo electrónico:

ferc015@racsa.co.cr

ISSN 0001-6002/2005/47/3/109-117 Acta Médica Costarricense, $@ 2005$ Colegio de Médicos y Cirujanos

\section{Resumen}

Actualmente se considera que la hipertensión arterial esencial es un verdadero problema de salud pública. Se estima que 1/6 de la población mundial sufre la enfermedad y en algunas poblaciones susceptibles la prevalencia es tan alta que 1 de 4 adultos mayores de 18 años es hipertenso. Aparentemente en Costa Rica la situación no es diferente a lo que ocurre mundialmente, se estima que hasta un $15.0 \%$ de los costarricenses son hipertensos; sin embargo, los reportes de prevalencia no se encuentran actualizados. No está clara la patogenia de la hipertensión arterial esencial y las hipótesis de que sus orígenes radicaban en el riñón y en el corazón no son válidas actualmente, debido a la evidencia que demuestra que esta es una enfermedad propia del endotelio vascular en donde se conjugan factores genéticos, ambientales, humorales e inflamatorios del vaso sanguíneo que inducen a disfunción vascular. Se discuten aspectos epidemiológicos y genéticos, enfatizando en el rol patogénico de los genes de angiotensina II, oxido nítrico y endotelina.
\end{abstract}

Descriptores: Hipertensión arterial, epidemiología, patogenia, genes, angiotensina II, óxido nítrico, endotelina.

Key words: Hypertension, epidemiology, patogenesis, genes, angiotesin II, nitric oxide, endothelin.

Recibido: 15 de febrero de 2005 Aceptado: 24 de mayo de 2005

\section{Generalidades y epidemiología}

La hipertensión arterial esencial (HTA) se ha transformado en los últimos años en un verdadero problema de salud pública. Actualmente se define HTA en los adultos mayores de 18 años de acuerdo a las directrices del Séptimo Reporte del Comité Nacional Conjunto (JNC VII, cuadro 1)1. Sin embargo, hay algunas diferencias cuando se comparan con las guías europeas (cuadro 2$)^{2}$.

Se estima que a nivel mundial hay cerca de 1,000 millones de personas afectadas por HTA y que la enfermedad afecta a 1 de cada 4 adultos mayores de 18 años en la población estadounidense ${ }^{3}$. En 1994 se estimaba que habían 50 millones de personas con HTA en los Estados Unidos. Sin embargo, de acuerdo a datos del (NHANES III) para el año 2002 ahora son 65 millones de personas, un aumento en la prevalencia de un $31.3 \%{ }^{4}$. En un reporte reciente de Kearney y colaboradores para el Instituto Nacional del Corazón, Pulmón y Sangre de los Institutos Nacionales de Salud (NHLBINIH) después de revisar y agrupar por regiones los reportes de diferentes países se estima que en el año 2000 un $26.4 \%$ de la población mundial 
tenía HTA, afectando a ambos géneros en forma muy similar, y se espera que para el año 2025 el $29.2 \%$ de la población mundial tendrá HTA lo que significará un incremento del $60.0 \%$ de personas con HTA (cuadro 3$)^{5}$. En series epidemiológicas se determina que la prevalencia de la enfermedad es mayor entre las personas de la raza negra y los individuos mayores, especialmente entre las mujeres, aproximadamente 35 millones de mujeres y 30 millones de hombres tienen HTA en los Estados Unidos.

En Costa Rica la prevalencia de la enfermedad no está bien definida y son pocos los estudios que se han publicado. En un estudio realizado por Arguedas y colaboradores en 1979 (Epidemilogía de la hipertensión arterial en Costa Rica. Estudio cooperativo 1976-1979; manuscrito no publicado,) se estableció que la prevalencia era de un $9.0 \%$, lo

\section{Cuadro 1. Definición de Hipertensión Arterial} de acuerdo al JNC VII1*

\begin{tabular}{|lcc|}
\hline $\begin{array}{l}\text { Clasificación de } \\
\text { Presión Arterial }\end{array}$ & $\begin{array}{c}\text { PA sistólica } \\
\text { (mmHg) }\end{array}$ & $\begin{array}{c}\text { PA diastólica } \\
\text { (mmHg) }\end{array}$ \\
\hline Normal & $<120$ & $<80$ \\
Pre-Hipertensión & $120-139$ & $80-89$ \\
Estadio 1 & $140-159$ & $90-99$ \\
Estadio 2 & $\geq 160$ & $\geq 100$ \\
Diabéticos + & $\geq 135$ & $\geq 85$ \\
\hline
\end{tabular}

Establecida para adultos mayores de 18 años de ambos sexos + Definido para diabéticos tipo 1 y tipo 2 .

\begin{tabular}{|lccccc|}
\hline \multicolumn{5}{|c}{ Cuadro 2. Guías Europeas para definición, clasificación y estadíos de riesgo cardiovascular en } \\
hipertensión arterial
\end{tabular}

que significaba que había un total 86,070 costarricenses hipertensos a esa fecha. En el estudio de la población de Zarcero realizado también por Arguedas y colaboradores ${ }^{6}$ realizado en la misma fecha se estableció la prevalencia en un $24.8 \%$, datos muy diferentes a los del estudio anterior y no se dan explicaciones sobre estas diferencias.

Es muy importante mencionar que en ambos estudios realizados por Arguedas se tomaron como referencia las cifras establecidas por la Organización Mundial de la Salud (OMS) que definían HTA a las personas con cifras mayores de $160 \mathrm{mmHg}$ de presión arterial sistólica y de $95 \mathrm{mmHg}$ de presión arterial diastólica. Considerando actualmente las cifras que definen la enfermedad es de esperar que la prevalencia sea mucho mayor de lo que fue reportado.
En 1999, Laclé reporta en un estudio en el cantón de Desamparados una prevalencia de un $15.4 \%$ en personas mayores de 15 años definiendo a HTA con cifras mayores de $140 / 90^{7}$.

En 1989 Ulate-Montero en una muestra de 934 niños y adolescentes de 6 a 16 años de seis instituciones educativas de Heredia y San José, reporta que la prevalencia de HTA en esta población es de $5.1 \%$ de acuerdo al percentilo 95 ajustado para género y edad y de un $3.4 \%$ de acuerdo a criterios de definición de HTA publicados en 1980 por Muñoz y colaboradores ${ }^{8,9}$.

La situación a futuro es más pesimista y preocupante cuando se establece el riesgo a largo plazo de desarrollar y sufrir HTA. En un estudio prospectivo de una cohorte de 


\begin{tabular}{|c|c|c|c|}
\hline \multicolumn{4}{|c|}{$\begin{array}{c}\text { Cuadro 3. Impacto global de hipertensión arterial en la población adulta y número total estimado } \\
\text { de adultos con hipertensión arterial }\end{array}$} \\
\hline Año & Total, \% (95\% Cl) & Hombres, \% (95\% Cl) & Mujeres, \% (95 \% Cl) \\
\hline 2000 & $\begin{array}{c}26.4 \\
(26.0-26.8)\end{array}$ & $\begin{array}{c}26.6 \\
(26.0-27.2)\end{array}$ & $\begin{array}{c}26.1 \\
(25.5-26.6)\end{array}$ \\
\hline \multirow[t]{2}{*}{2025} & $\begin{array}{c}29.2 \\
(28.8-29.7)\end{array}$ & $\begin{array}{c}29.0 \\
(28.6-29.4)\end{array}$ & $\begin{array}{c}29.5 \\
(29.1-29.9)\end{array}$ \\
\hline & & & n $(95 \% \mathrm{Cl})$ \\
\hline \multicolumn{3}{|c|}{ Número total mundial en el 2002} & $\begin{array}{l}972 \text { millones } \\
(957-987)\end{array}$ \\
\hline \multicolumn{3}{|c|}{ Número total en países económicamente desarrollados en el 2000} & $\begin{array}{l}333 \text { millones } \\
(329-336)\end{array}$ \\
\hline \multicolumn{3}{|c|}{ Número total en países en vías de desarrollo en el 2000} & $\begin{array}{l}639 \text { millones } \\
(625-654)\end{array}$ \\
\hline \multicolumn{3}{|c|}{ Número total estimado mundial en el 2025} & $\begin{array}{c}1560 \text { millones } \\
(1.54-1.58)\end{array}$ \\
\hline
\end{tabular}

1,298 participantes con edades que oscilaban entre los $55 \mathrm{y}$ 65 años de edad y que se encontraban libres de HTA al inicio del estudio, se determinó que su riesgo residual para desarrollar HTA en cualquier momento de la vida fue de un $90 \%{ }^{10}$. Ante este reporte, deberá esperarse un aumento sustancial e importante de las personas con HTA, no solamente por el incremento en la expectativa de vida de la población sino también por el riesgo desarrollar la enfermedad.

Ahora es bien reconocido que esta es una enfermedad que afecta no solamente a los adultos, sino que también a los niños y adolescentes.

Estudios epidemiológicos han determinado que existe un vínculo entre la elevación de la presión arterial en niños y adolescentes y el riesgo de desarrollar HTA en la edad adulta ${ }^{11,12}$. Aunque en este grupo es más frecuente encontrar HTA por causa secundaria, también se presenta HTA esencial y se considera que la prevalencia de la enfermedad está incrementándose en niños, pero especialmente entre el grupo de los adolescentes. Los reportes epide-miológicos aportan valores de prevalencia muy variables. En datos revisados por Rosner y colaboradores de 1978 a 1991 se lograron obtener las cifras de la presión arterial sistólica (PAS) en 47,196 niños y presión arterial diastólica (PAD) en 38,184 tomando en cuenta el quinto ruido de la fase de Korotkoff y se demostró una prevalencia general de $4.4 \%$ de acuerdo a la PAS y de $3.2 \%$ para la PAD ${ }^{13}$. En otro grupo de niños de 10 a 15 años de edad que cursaban entre el $5^{\text {to }}$ y $8^{\text {vo }}$ año de escuela de las escuelas públicas de Saint Paul y Minneapolis, se obtuvo el nivel de presión arterial de
14, 686 sujetos de ambos géneros (3,394 de raza negra y de 11,292 de raza blanca), se reporta una prevalencia general de $2.7 \%$ y $2.0 \%$ de acuerdo a la PAS y PAD. En este mismo reporte se determinó que la prevalencia global de HTA sistólica es ligeramente mayor y la prevalencia de HTA diastólica era ligeramente menor en comparación a los datos de $1987^{14}$.

En recomendaciones actuales la Sociedad Americana del Corazón (AHA) está siguiendo las directrices del Segundo Grupo de Trabajo del Instituto Nacional del corazón, pulmón y sangre (NHLBI) sobre presión arterial en donde se establece que a todos los niños mayores de 3 años se les debe de medir la presión arterial durante cualquier evaluación rutinaria. Incluso el Programa de Educación Nacional sobre Hipertensión Arterial (NHBPEP) recomienda que se utilicen los estándares de presión arterial de acuerdo a edad, género y talla en niños mayores de 1 año y en adolescentes. Se hace notar en la recomendación específica que la toma de la presión arterial no es diferente a la establecida en adultos y por lo tanto debe de realizarse con un esfingomanómetro de mercurio o con uno aneroide bien calibrado y que debe de tomarse la presión arterial en el brazo derecho, con el niño sentado y con el brazo descansando sobre una superficie sólida que se encuentre a nivel del corazón ${ }^{16}$.

Está muy bien definido que bajar las cifras de presión arterial ya sea por medio de drogas antihipertensivas o por cambios en el estilo de la vida, disminuye significativamente la mortalidad y la morbilidad de origen cardiovascular. 
Diversos estudios epidemiológicos han demostrado que tanto la PAS como la PAD tienen una asociación estadística continua y fuertemente positiva con el estado de la enfermedad cardiovascular y de que existe riesgo cardiovascular a lo largo de cualquiera de los niveles de presión arterial ${ }^{17,18}$.

Strandberg observó a 3,267 hombres previamente sanos durante un período de 32 años y durante todo ese tiempo, la relación entre PAS y mortalidad se incrementó a partir del rango de 131-140 mmHg. En este estudio hubo 701 muertes, 234 (33.4\%) debidas a enfermedad arterial coronaria, 49 (7.0\%) a eventos vasculares cerebrales, $42(6.0 \%)$ a otras causas cardiovasculares y 204 (29.1\%) correlacionadas a cáncer ${ }^{19}$.

En el estudio Tratamiento Óptimo de la Hipertensión (HOT), 18,790 sujetos fueron asignados a alcanzar diferentes cifras de PAD. 6,262 sujetos a $\leq 80 \mathrm{mmHg}, 6,264 \mathrm{a} \leq 85$ y $6,264 \leq 90 \mathrm{mmHg}$ respectivamente. Se determinó que la menor incidencia de eventos cardiovasculares ocurre con cifras de PAD menores de $82 \mathrm{mmHg}$ y que el menor riesgo de mortalidad cardiovascular se obtiene con cifras de PAD menores a $86.5 \mathrm{mmHg}{ }^{20}$. Antes de la nueva clasificación del JNC VII se utilizaba la clasificación del JNC VI ${ }^{21}$ en donde se establecían difrentes categorías. En un análisis de 6,859 sujetos no hipertensos (3,892 mujeres y 2,967 hombres) catalogados como portadores de presión normal alta, Vasan y colaboradores establecen que la incidencia acumulada de enfermedad cardiovascular a los 10 años en todas las personas de 35 a 64 años es de $4.0 \%$ para las mujeres y de $8.0 \%$ para los hombres y para los sujetos mayores (aquellos de 65 a 90 años) es de $18.0 \%$ para las mujeres y $25.0 \%$ para los hombres ${ }^{22}$. Pareciera por lo tanto que para no incrementar la morbilidad y mortalidad de origen cardiovascular deben de mantenerse cifras de presión arterial más bajas de lo que actualmente están definidas como normales y se viene a confirmar que entre más baja se mantenga la presión arterial en los sujetos afectados por la enfermedad, menor es la morbilidad y mortalidad cardiovascular.

Ante esta evidencia cabe entonces preguntarse qué es presión arterial normal. Un metanálisis de 61 estudios observacionales prospectivos evaluó los registros individuales de 958,074 participantes. Basados en 56,000 muertes vasculares durante el período de seguimiento de 12.7 millones de personas-año, los autores de los estudios colaborativos prospectivos concluyen que la presión arterial normal debe ser de 115/75 o menos, ya que por debajo de estas cifras no hay correlación con mortalidad cardiovascular. Observaron además personas de edad media (40-69 años) que tienen un incremento de $20 \mathrm{mmHg}$ en la PAS y de $10 \mathrm{mmHg}$ de la PAD, experimentan un incremento de dos veces en el riesgo de muerte por evento vascular cerebral o por enfermedad coronaria ${ }^{23}$. Es de notar que este es el metanálisis más grande de su tipo que se ha realizado a la fecha.
Se conoce de las variaciones geográficas de la enfermedad vascular y su comportamiento y los factores de riesgo asociados en diversas partes del mundo. Se compararon las diferentes prevalencias en 6 países europeos con los de Norteamérica y se observó que la prevalencia es mayor en Alemania (55.0\%), Finlandia (49.0\%), España (47.0\%), Suecia $(38.0 \%)$ e Italia $(38.0 \%)$ comparados con Estados Unidos $(28.0 \%)$ y Canadá $(27.0 \%)$ respectivamente ${ }^{24}$.

A pesar de las diferentes cifras en prevalencia de HTA que se reportan tanto en niños, adolescentes y adultos, lo cierto es que esta enfermedad no es exclusiva de ningún grupo ni género en particular, de que existe un aumento en la prevalencia a nivel mundial y que intervenir terapéuticamente es una conducta médica adecuada, y que en un futuro no muy lejano, debemos esperar que el número de personas afectadas por la enfermedad se incremente en forma muy importante.

\section{Genes e hipertensión arterial}

La diversidad y variabilidad genética es un principio fundamental e implica diferentes susceptibilidades frente a los interminables estímulos externos e internos, así como su respuesta frente a la enfermedad. La presión arterial está regulada por un complejo sistema de procesos que influyen no solamente sobre el gasto cardíaco, sino también sobre el volumen de eyección, la frecuencia cardíaca, las resistencias vasculares periféricas y la capacitancia venosa ${ }^{25}$. Desde los aportes de Furchgott ${ }^{26}$, Palmer y Moncada ${ }^{27}$ en la década de los 80 sobre el papel del oxido nítrico en el fenómeno de relajación vascular se ha progresado mucho sobre la comprensión de la fisiología normal del vaso sanguíneo y su relación como órgano rector y blanco de múltiples condiciones clínicas. A diferencia de enfermedades genéticas típicamente definidas y caracterizadas por la presencia o ausencia de un gen específico, en la HTA se han demostrado la presencia de polimorfimos moduladores de diferentes funciones. Se han estudiado los polimorfismos del sistema renina-angiotensina-aldosterona (SRA), que constituyen el grupo más estudiado tanto en vivo como in vitro y se ha sugerido que existe una relación entre las funciones vasculares y la variabilidad genética de este sistema (Cuadro 4). Además, se han estudiado los polimorfismos del sistema de endotelina, oxido nítrico-cGMP (NO-cGMP) y sistemas oxidativos (NADPH oxidasa). En todas estas circunstancias se ha logrado documentar asociación con enfermedad debido a la alteración en la expresión genética y la modificación de la información para el funcionamiento del vaso sanguíneo. 


\begin{tabular}{|c|c|c|}
\hline \multicolumn{3}{|l|}{ Sistema adrenérgico } \\
\hline Alfa-2B & Deleción & Aumento de eventos coronarios \\
\hline Alfa-1 & Mutación/Gly 16 & Aumento hipertensión en pacientes diabéticos \\
\hline Beta-2 & Mutación/Arg492 & Sin relación con eventos cardiovasculares \\
\hline NADPH oxidasa & $\mathrm{C} 242 \mathrm{~T}$ & Aumento del riesgo de enfermedades cerebrovasculares \\
\hline eNOS (NOS III) & Glu298Asp & Disfunción endotelial? \\
\hline \multirow[t]{3}{*}{ ECA } & Deleción & $\begin{array}{l}\text { Aumento de contractilidad a la adrenalina } \\
\text { Aumento de la actividad de la ECAQ }\end{array}$ \\
\hline & & Aumento de contractilidad \\
\hline & & Aumento riesgo enfermedades cardiovasculares \\
\hline Angiotensinógeno & M235T & Aumento de niveles plasmáticos de angiotensinógeno \\
\hline Preproendotelina & Lys198Asn & Aumento de la contractilidad \\
\hline \multicolumn{3}{|c|}{$\begin{array}{l}\text { Condiciones genéticas que por mutaciones, deleciones o sustituciones sobre un gen específico que controlan la reactividad vascular y } \\
\text { su efecto en estudios clínicos. A pesar de que se han descrito muchos otros polimorfismos, se anotan los que se han demostrado que } \\
\text { influyen claramente en la homeostasis vascular. (Adaptado de Henrion29) NAPDPH: Nicotín adenín dinucleótido. eNOS: Oxido nítrico } \\
\text { sintetasa endotelial ECA: Enzima convertidora de angiotensina }\end{array}$} \\
\hline
\end{tabular}

\section{Angiotensina II}

La AII, cuyo efecto principal es aumentar la amplificación del tono contráctil vascular, participa activamente en fenómenos relacionados con el crecimiento celular, hipertrofia muscular, inducción inflamatoria, oxidación lipoproteica, fibrosis intersticial y activación plaquetaria entre otros ${ }^{28-31}$. El polimorfismo más estudiado es el de la enzima convertidora de angiotensina (ECA) en sus formas de inserción o deleción. Se ha demostrado que cuando existe sobre-expresión del gen que controla la actividad de la ECA y consecuentemente AII, hay un aumento plasmático y cardíaco de la actividad de la $\mathrm{ECA}^{32}$ una mayor respuesta contráctil de la arteria mamaria interna frente a Angiotensina I in vitro ${ }^{33,34}$, e incremento en el grosor de la pared carotídea ${ }^{35}$. Asimismo los efectos inflamatorios, mitogénicos, oxidativos y apoptóticos se ven exacerbados cuando se incrementa la actividad de la ECA ${ }^{36,37}$. Después de la descripción Rigat ${ }^{38}$ en 1990 de un polimorfismo inserción/deleción para el gen de la ECA se conoce ahora que los niveles de la ECA son mayores en individuos homocigotos para el alelo D e intermedios en los sujetos heterocigotos. Este alelo ha sido asociado con diversas condiciones mórbidas en los que el SRA está involucrado, incluyendo infarto agudo al miocardio (IAM) en personas de bajo riesgo, hipertrofia ventricular izquierda y progresión de nefropatía diabética ${ }^{39,40}$. Sin embargo, este polimorfismo no ha sido asociado consistentemente con el desarrollo de HTA, prevalencia de enfermedad coronaria, extensión de la enfermedad coronaria o IAM ${ }^{41}$.
En una muestra de 1109 mujeres gemelas dizigóticas del Reino Unido estudiadas por ampliación genómica por deLange y colaboradores se logró replicar un vínculo en el cromosoma 11 que influencia el control de la presión arterial sistólica y diastólica, pero más la sistólica, sugiriendo que puede ser un candidato genético potencial para explicar la influencia que ejerce no solamente la herencia sino el medio ambiente y el estilo de vida en el control de la presión arterial ${ }^{42}$.

A pesar de todos los efectos que se han reconocido que tiene AII sobre el vaso sanguíneo, aún no se ha logrado aclarar la forma en que las diversas expresiones genéticas interactúan con la herencia, el medio ambiente, la dieta y el estilo de vida para iniciar el proceso de enfermedad. Esperamos que en un futuro todas estas dudas se aclaren, especialmente con los estudios actuales que se realizan en el genoma humano.

\section{Óxido Nítrico}

La síntesis de ON (óxido nítrico) en el endotelio vascular es esencial para la regulación del tono vascular y el control de la presión arterial tanto en animales como en seres humanos. Se ha logrado demostrar que ocurre HTA esencial 3.8 veces más frecuente en personas menores de 50 años que tienen dos o más parientes de primer grado que han desarrollado HTA antes de los 55 años, y los hijos de sujetos hipertensos tienen reducida la respuesta vasodilatadora 
estimulada por acetilcolina, sugiriendo que hay una importante contribución genética al desarrollo de la enfermedad y que la síntesis y vía metabólica del NO se encuentra alterada ${ }^{43}$ por lo que la vasodilatación dependiente del endotelio puede verse afectada por los polimorfismos. Existe una intrincada relación entre el óxido nítrico, el stress oxidativo, AII, inflamación vascular y factores de riesgo, y a la fecha no se han logrado aclarar muchos de los aspectos involucrados en la génesis de la enfermedad hipertensiva y la respuesta hipertensiva vascular (Figura 1) ${ }^{44-46}$. Factores genéticos, humorales y hemodi-námicos se conjugan para generar de diversas formas una mayor actividad de la
NADPH oxidasa produciendo mayor cantidad de radicales libres de oxígeno, peróxido de hidrógeno, peroxinitrito y disminuyendo la cantidad disponible de óxido nítrico para inducir disfunción endotelial e hipertrofia de la media del vaso (figura 2). Se han planteado en múltiples publicaciones que el control genético de la vía metabólica del ON y la mutación y varianza de los genes que controlan esta vía, están directamente involucradas en el desarrollo de HTA y otras complicaciones de tipo cardiovascular. El metabolismo del oxígeno celular genera especies de oxígeno potencialmente deletéreas, el riñón y la vasculatura son fuentes importantes de actividad de la NADPH, que bajo

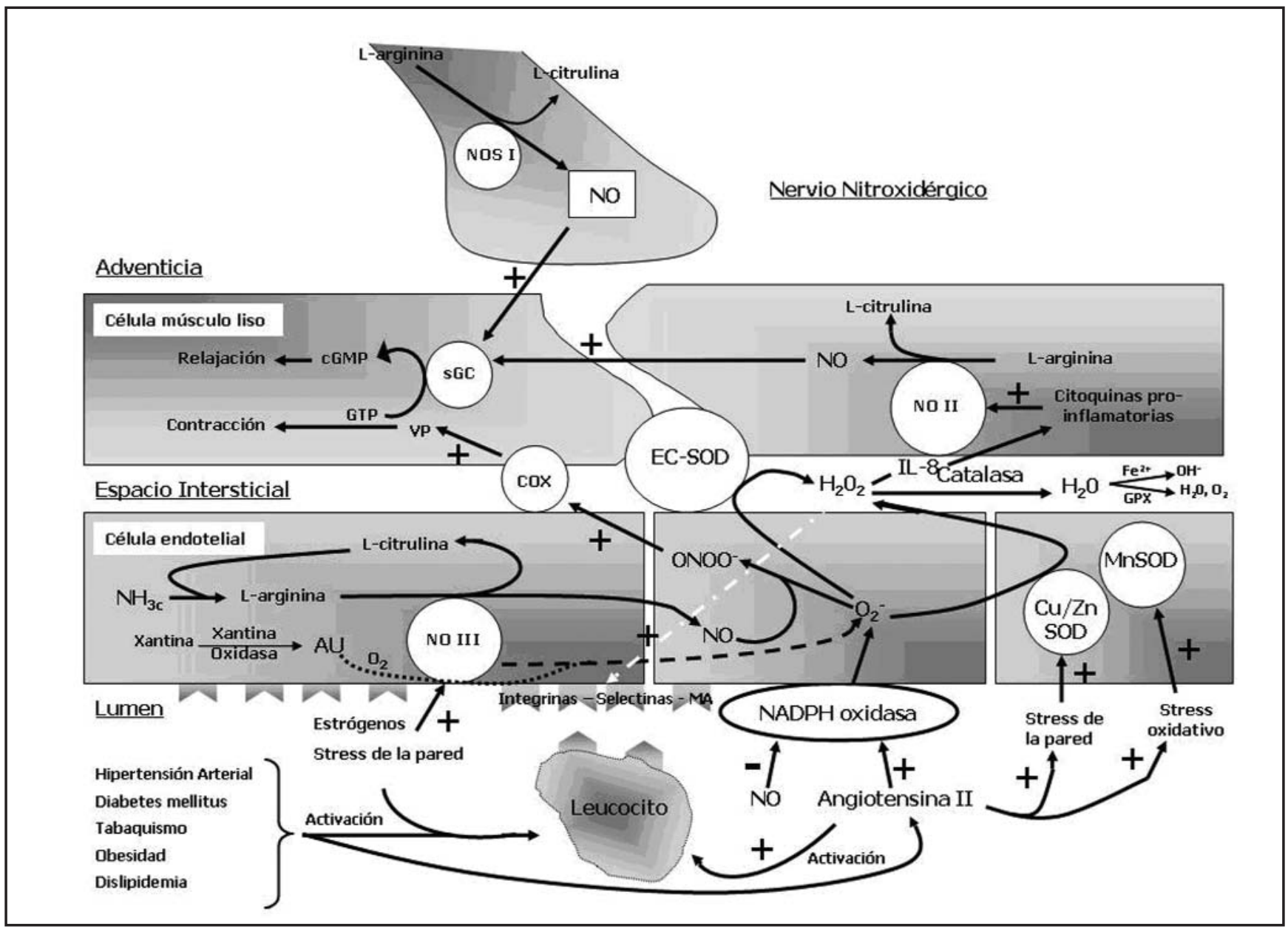

Figura 1. Relación entre oxido nítrico, anión superóxido, oxidación e inflamación vascular

Algunas de las interacciones complejas involucradas en la regulación del balance oxido nítrico (NO) y anión superóxido $\left(\mathrm{O}_{2}{ }^{-}\right)$en el vaso sanguíneo y su relación con algunos de los fenómenos inflamatorios desencadenados por factores de riesgo. Las enzimas antioxidantes superóxido dismutasa (SOD, catalasa) previenen el stress oxidativo. El NO actúa a través de mecanismos dependientes e independientes de transcripción para prevenir la adhesión leucocitaria, plaquetaria y la inflamación vascular. El peróxido de hidrógeno con su poder oxidante, estimula la mayor expresión y actividad de integrinas, selectinas y moléculas de adhesión de la célula endotelial, facilitando la adhesión y migración de células inflamatorias. El ácido úrico cuando interactúa con el oxígeno produce mayor cantidad de radicales libres de oxígeno y el agua libre cuando entra en contacto con el hierro genera radicales hidroxilo. Los factores de riesgo producen activación de enzimas que incrementan la producción de grandes cantidades de anión superoxido. NOS I: Óxido nítrico sintetasa neuronal. NOS II: óxido nítrico sintetasa inducible. NOS III: óxido nítrico sintetasa endotelial. EC-SOD: superóxido dismutasa extracelular. MN-SOD: superóxido dismutasa manganésica. $\mathrm{Cu} / \mathrm{Zn}$ SO: superóxido dismutasa cobre/zinc. SGC: guanilato ciclasa soluble. ONOO-: peroxinitrito. $\mathrm{H}_{2} \mathrm{O}_{2}$ : peróxido de hidrógeno. GTP: guanosin-5-trifosfato. COX: ciclooxigenasa. MA: moléculas de adhesión VP: prostanoides vasoconstrictores. AU: ácido úrico. GPX: glutation peroxidasa Adapatado de Mclntyre M y Granger ${ }^{44,45}$ 


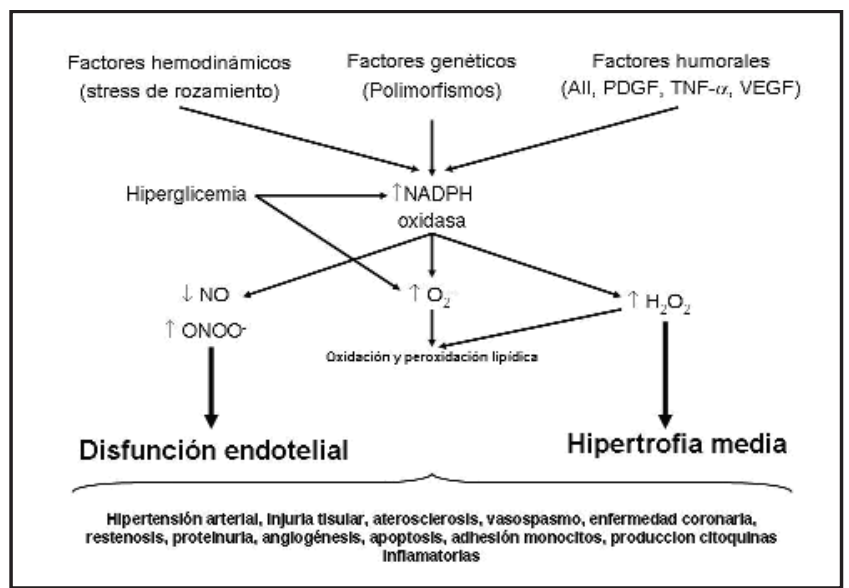

Figura 2. Relación entre factores genéticos, hemodinámicos y humorales y la inducción de $\mathrm{O}_{2}{ }^{-}$

La activación de la NADPH genera cambios y consecuencias en diferentes condiciones, particularmente en HTA. Pueden estar involucrados cambios genéticos en la modulación de la expresión de los componentes del sistema NADPH. Se disminuye la biodisponibilidad del NO debido a una mayor actividad de la NADPH que genera anión superóxido formando un círculo vicioso que produce finalmente un desbalance entre sustancias vasodilatadoras y vasoconstrictoras. Finalmente sobreviene disfunción endotelial. Los factores de crecimiento interactúan con radicales libres de oxígeno para inducir no solamente disfunción endotelial sino también crecimiento celular e hipertrofia de la media del vaso.

All: Angiotensina II PDGF: Platelet derived growth factor (factor de crecimiento derivado de las plaquetas) TNF- $\alpha$ : Tumor necrosis factor $\alpha$ (factor de necrosis tumoral) VEGF: Vascular endothelial growth factor (factor de crecimiento derivado del endotelio vascular) NO: Óxido nítrico ONOO-: Peroxinitrito $\mathrm{O}_{2}{ }^{-}$: Radical libre de oxígeno, anión superóxido $\mathrm{H}_{2} \mathrm{O}_{2}$ : Peróxido de hidrógeno. Adaptado de Zalba y colaboradores. ${ }^{46}$

condiciones patológicas juega un papel importante en la disfunción renal y el daño vascular. Miyamoto y colaboradores estudiaron una población de 879 sujetos de las ciudades de Kyoto y Kumanoto (240 y 223 normotensos y 218 y 187 hipertensos respectivamente) demostrando que al menos en esa población existe una variante de codificación del gen eNOS/Glu298Asp (oxido nítrico sintetasa endotelial/glutamina298aspartato) que muestra una fuerte asociación con HTA, sugiriendo de que se trata de un factor genético de susceptibilidad para el desarrollo de HTA ${ }^{47}$.

Lacy y colaboradores examinaron el papel de la herencia que tiene la producción de peróxido de hidrógeno $\left(\mathrm{H}_{2} \mathrm{O}_{2}\right)$ en una cohorte familiar de 236 sujetos con hipertensión y lograron determinar que la producción de $\mathrm{H}_{2} \mathrm{O}_{2}$ se correlaciona con la actividad de la renina plasmática, sugiriendo que hay una importante interacción entre los niveles circulantes de radicales de oxígeno y el sistema renina-angiotensina. Además, lograron demostrar que la pro ducción de $\mathrm{H}_{2} \mathrm{O}_{2}$ se correlaciona negativamente con la contractilidad cardíaca y la función renal y hasta un $35.0 \%$ de la varianza observada en la producción de $\mathrm{H}_{2} \mathrm{O}_{2}$ podría ser atribuida a factores genéticos, sugiriendo que hay un componente hereditario que determina la lesión a órgano blanco en HTA $^{48}$.

\section{Endotelina}

De todas las sustancias vasoconstrictoras probablemente la endotelina sea la más potente. Ahora se reconocen factores que estimulan la mayor liberación de endotelina tales como niveles elevados de LDL oxidados, hiperglicemia, deficiencia estrogénica, obesidad, uso de cocaína, edad y factores procoagulantes tales como la trombina, sin dejar de mencionar a factores de crecimiento, citoquinas y moléculas de adhesión ${ }^{49}$.

Se ha descrito un polimorfismo de la preproendotelina-1 (transversión G/T en la posición 595 y una transversión Lys/Asn en la posición 198) que va asociada con mayores niveles de presión arterial en pacientes con sobrepeso que son portadores del alelo $\mathrm{T}^{50}$.

No está muy clara la participación de endotelina en la HTA, ya que sujetos hipertensos sal sensibles usualmente tienen bajos niveles de renina y los niveles de endotelina en el plasma responden en una forma exagerada, incrementándose después de la depleción de sodio con incremento concomintante de las catecolaminas en el plasma ${ }^{51}$. Esto sugiere una relación entre el sistema simpático, la sensibilidad al sodio y la reactividad del sistema de endotelina que podría contribuir a la elevación de la presión arterial en estos sujetos ${ }^{52}$.

La relación entre el sistema de endotelina y angiotensina y óxido nítrico es compleja y su participación en la fisiopatología de la HTA aún no está claramente definida. Los dos sistemas, endotelina y angiotensina, pueden actuar más bien en forma paralela que en forma lineal y esto podría tener implicaciones terapéuticas para la asociación potencial de drogas que intervengan bloqueando el sistema renina-angiotensina y bloqueadores del receptor de endotelina. Esto podría finalmente resultar en una mayor reducción de la presión arterial al bloquear a los dos sistemas vasoconstrictores más potentes conocidos a la fecha y podría tener una eventual repercusión benéfica en el daño y lesión a órgano blanco inducido por HTA.

El fenotipo final de hipertensión arterial podría ser similar entre las personas, pero las determinantes hereditarias subyacentes para la elevación de la presión arterial puede que sean poligénicas y heterogéneas. Las antiguas teorías de que la génesis de la HTA reside en el control renal inadecuado del volumen sanguíneo, del agua y del sodio y en el desbalance de los mecanismos de control autonómico y cardíaco, han pasado a la historia con las evidencias y 
teorías modernas de que esta es una enfermedad propia del endotelio vascular con un fuerte trasfondo genético. Aunque aún no se han logrado dilucidar cuáles son todas esas determinantes e interacciones, cada día se encuentran otros genes, moléculas y sustancias involucradas no solamente en el control de la presión arterial, sino también con la aparición y severidad de la enfermedad y lesión a órgano blanco.

La literatura a lo largo del tiempo y a la fecha ha logrado establecer que la HTA es un trastorno con un rasgo genético múltiple que resulta de una compleja interacción entre el medio ambiente y la herencia, lo que hace más difícil comprender la etiopatogenia de esta enfermedad. Se espera que con el estudio detallado del genoma humano puedan aclarase muchas de las dudas que existen actualmente y que finalmente pueda encontrarse el o los genes involucrados no solamente en la génesis de la HTA sino también en el desarrollo de la enfermedad, lesión a órgano blanco y complicaciones de la misma. Esta comprensión genética finalmente puede ofrecer nuevas alternativas de tratamiento que realmente libren al ser humano de sufrir HTA y sus complicaciones.

\section{Abstract}

It is considered actually that high blood pressure represents a real public health problem. It is estimated that $1 / 6$ of the world have high blood pressure and in some susceptible populations the prevalence it's as high as much as 1 of 4 adults over 18 years old suffers from high blood pressure. In Costa Rica the situation is not different of what happens worldwide and is estimated that $15.0 \%$ of the costarrican population have high blood pressure although the prevalence data are not actualized. The pathogenesis of essential high blood pressure remains unclear and the hypothesis that their origins were on the kidney and the heart are no longer valid because actual evidence demonstrates that this is a disease of the vascular endothelium where genetic, ambiental, humoral and inflammatory factors of the blood vessels induce vascular dysfunction. We discuss epidemiologic and genetic factors emphasizing on the pathogenic role of the angiotensin II, nitric oxide and endothelin genes.

\section{Referencias}

1. Joint National Comitee on Prevention, Detection, Evaluation, and Treatment of High Blood Pressure. The seventh report of the Joint National Committee on Prevention, Detection, Evaluation, and Treatment of High Blood Pressure. JAMA 289; 19: 2560 - 2572

2. 2003 European Society of Hypertension - European Society of Cardiology guidelines for the management of arterial hypertension. Guidelines Committee. J Hypertens 2003; 21: 1011 - 1053

3. Burt VL, Cutler JA, Higgins M, Labarthe D, Whelton P, Brown C, Rocella EJ. Trends in the prevalence, awareness, treatment and control of hipertensión in the adult US population: data from the health examination surveys, 1960 to 1991. Hypertension 1995; 26: 60 - 69

4. Fields LE, Burt VL, Cutler JA, Hughes J, Rocella E, Sorlie P. The Burden of Adult Hypertension in the United States 1999 to 2000. A Rising Tide. Hypertension 2004; 44: 398 - 404

5. Kearney PM, Whelton M, Reynolds K, Muntner P, Whelton PK, He J. Global burden of hypertension: analysis of worldwide data. Lancet 2005; 365: $217-23$.

6. Arguedas-Chaverri C, Vargas-Martínez H, Soto-Arroyo VM, VargasSánchez YM, Vega Méndez MA. Estudio de la prevalencia de hipertensión arterial en Zarcero de Alfaro Ruiz, provincia de Alajuela. Act Med Cost 1982; 25: 315 - 321.

7. Laclé-Murray, A. Diagnóstico y control de la hipertensión arterial en el adulto mayor (comparación urbana/rural) Acta Méd Costarric 1999; 41:15-21.

8. Ulate-Montero, G. Valores de presión arterial en una muestra de niños y adolescentes del valle central de Costa Rica. Acta Méd Costarric 1989; $33: 83-88$.

9. Muñoz S, Muñoz H, Zambrano F. Blood pressure in a school age population. Mayo Clin Proc 1980; 55:623 - 632.

10. Vasan RS, Beiser A, Larson M, Kannel W, D`Agostino R, Levy D. Residual Lifetime Risk for Developing Hypertension in Middle-aged Women and Men. The Framingham Heart Study. JAMA 2002; 287: $1003-1010$.

11. Luepker RV, Jacobs DR, Prineas RJ, Sinaiko AR. Secular trends of blood pressure and body size ina a multi-ethnic adolescent population: 1986 - 1996. J Pediatr 1999; 134: 668 - 74.

12. Buzzard CJ, Lipshultz SE. High blood pressure in infants, children, and adolescents. In Hoekelman RA, editor. Primary pediatric care. 4th ed. St Louis: Mosby; 2001. p. 1142 - 9.

13. Rosner B, Prineas R, Daniels SR, Loggie J. Blood pressure differences between blacks and whites in relation to body size among US children and adolescents. Am J Epidemiol 2000; 151: 1007 - 1019.

14. Adrogué H, Sinaiko AR. Prevalence of Hypertension in Junior High School-Aged Chinldren: Effect of New Recommendations in the 1996 Updated Task Force Report. Am J Hypertens 2001; 14: 412414.

15. Williams ChL, Hayman L, Daniels S, Robinson T, Steinberg J, Paridon S, Bazzarre T. A Statement for Health Proffesionals From the Committee on Atherosclerosis, Hypertension, and Obesity in the Young (AHOY) of the Council on Cardiovascular Disease in the Young, American Heart Association. Circulation 2002; 106: 143 - 160.

16. Stamler J, Stamler R, Neaton JD. Blood pressure, systolic and diastolic, and cardiovascular risks: US population data. Arch Intern Med 1993; $153: 598-615$.

17. Kannel WB. Blood pressure as a cardiovascular risk factor: prevention and treatment. JAM 1996; 275: 1571 - 6 .

18. van den Hoogen PCW, Feskens EJM, Nagelkerke NJD, Menotti A, Nissinen A, Kromhout D. The relation between blood pressure and mortality due to coronary heart disease among men in differents parts of the world. N Engl J Med 2000; 342: 1 - 8 . 
19. Strandberg TE, Salomaa VV, Vanhanen HT Pitkälä K. Blood pressure and mortality during a 32-year-follow-up. J Hypertens 2001; 19: $35-39$.

20. Hansson L, Zanchetti A, Carruthers SG, Dahlöf B, Elmfeldt D, Julios $\mathrm{S}$, Ménard J, Rahn KH, Wedel H, Westerling S. Effects of intensive blood-pressure lowering and low-dose aspirin in patients with hypertension: principal results of the Hypertension Optimal Treatment (HOT) randomized trial. Lancet 1998; 351: 1755 - 62.

21. Joint National Comitee on Prevention, Detection, Evaluation, and Treatment of High Blood Pressure. The sixth report of the Joint National Committee on Prevention, Detection, Evaluation, and Treatment of High Blood Pressure. JNC VI. Arch Intern Med 1997; 155: $2413-46$.

22. Vasan RS, Larson MG, Leip EP, Evans JC, O'Donnell CJ, Kannel WB, Levy D. Impact of high-normal blood pressure on the risk of cardiovascular disease. N Engl J Med 2001; 345: 1291 - 7.

23. Lew;ngton S, Clarke R, Qizilbash N, Peto R, Collins R. Age-specific relevance of usual blood pressure to vascular mortality: a metaanalysis of individual data for one million adults in 61 prospective studies. Lancet 2002; 360: 1903 - 1913.

24. Wolf-Maier, K, Cooper RS, Banegas JR, Giampaoli S, Hense HW, Joffres $\mathrm{M}$ et al. Hypertension Prevalence and Blood Pressure Levels in 6 European Countries, Canada and the United States. JAMA 2003; 298: 2363 - 2369

25. Bakris GL, Mensah GA. Pathogenesis and Clinical Physiology of Hypertension. Curr Probl Cardiol 2003; 28: 137 - 155.

26. Furgchtott RF, Zawadzki JV. The obligatory role of endothelial cells in the relaxation or arterial smooth muscle by acetylcholine. Nature 1980; 288: 373 - 386 .

27. Palmer RMJ, Ferrige AG, Moncada S. Nitric oxide accounts for the biological activity of endothelium - derived relaxing factor. Nature 1987; 327: $524-526$.

28. Lyons D, Webster J, Benjamin N. Angiotensin II. Adrenergic sympathetic constrictor action in humans. Circulation 1995; 91: 1457 1460 .

29. Henrion D, Laher I, Laporte R, Bevan JA. Angiotensin II amplifies arterial contractile response to norepinephrine without increasing $\mathrm{Ca}++$ influx: role of protein kinase C. J Pharmacol Exp Ther 1992; 261: $835-40$.

30. Gaedeke J, Noble N, Border W. Angiotensina II e insuficiencia renal progresiva. Curr Hypertens Repor Ed Esp 2003; 2: 47 - 52.

31. Ruiz-Ortega M, Lorenzo O, Rupérez M, Estaban V, Suzuki Y, Mezzano S, Plaza JJ, Egido J. Role of the Renin-Angiotensin System in Vascular Disease. Expanding the Field. Hypertension 2001; 38: $1382-1387$.

32. Danser AH, Schalekamp MA, Bax WA, van den Brink AM, Saxena $\mathrm{PR}$, Riegger GAJ et al. Angiotensin-converting-enzyme in the human heart. Effect of the deletion/insertion polymorphism. Circulation 1995; 92: $1387-1388$.

33. Ueda S, Elliot HL, Morton JJ, Connel JMC. Enhanced pressor response to angiotensin $\mathrm{I}$ in normotensive men with the deletion genotype (DD) for angiotensin-converting enzyme. Hypertension 1995; 25 : 1266 - 1269 .

34. Castellano M, Muiesan ML, Rizzoni D, Beschi M, Pasini G, Cinelli A et al. Angiontensin-converting enzyme I/D polymorphism and arterial wall thickness in a general population. The Vorbano Study. Circulation 1995; 91: 2721 - 2724.

35. Chadwichk IG, O'Toole L, Morice AH, Wilfred YW, Jackson PR, Ramsay LE. Pressor and hormonal responses to angiotensin I infusion in healthy subjects of different angiontensin-converting enzyme genotypes. J Cardiovasc Pharmacol 1997; 29: 485 - 489.

36. Ruiz-Ortega M, Lorenzo O, Suzuki Y, Ruperez M, Egido J. Proinflammatory actions of angiotensin II. Curr Opin Nephrol Hypertens 2001; 10: 321 - 329.
37. Zhou M-S, Schulman I, Raij L. Nitric Oxide, Angiotensin II and Hypertension. Sem in Nephrol 2004; 24: 366 - 378.

38. Rigat B, Hubert C, Alhenc-Gelas F, Cambien F, Corvol P Soubrier F. An insertion/deletion polymorphism in the angiotensin I-converting enzyme gene accounting for half the variance of serum enzyme levels. J Clin Invest 1990; 86: 1343 - 1346.

39. Sharma P, Fatibene J, Ferraro F, Jia H, Monteith S, Brown C et al. A genome-wide search for susceptibility loci to human essential hypertension. Hypertension 2000; 35: 1291 - 1296

40. Dzau VJ, Bernstein K, Celermajer D, Cohen J, Dahlöf B, Deanfield J et al. The relevance of tissue angiotensin-converting enzyme: manifestations in mechanistic and endpoint data. Am J Cardiol 2001; 88(suppl): 1L-20L

41. Pfohl M, Koch M, Prescod S, Haase KK, Haring HU, Karsch KR. Angiotensin I-converting enzyme polymorphism, coronary artery disease and myocardial infarction: an angiographically controlled study. Eur Heart J 1999; 20: 1318 - 1325

42. de Lange M, Spector T, Andrew T. Genome-wide scan for blood pressure suggest linkage to chromosome 11, and replication of loci on 16, 17, and 22. Hypertension 2004; 44: $872-877$

43. Williams RR, Hunt SC, Hasstedt SJ, Hopkins PN, Wu LL, Berry TD, Stults BM, Barlow GK, Schumacher MC, Lifton RP, Lalouel JM. Are the interactions between genetic and environmental factors predisposing to high blood pressure? Hypertension 1991; 18(suppl I): I29I37

44. McIntyre M, Bhor D, Dominiczak A. Endotelial function in hipertensión. The role of the superoxide anion. Hipertensión 1999; 34: 539 $-545$

45. Granger DN, Vowinkel T, Petnehazy. Modulation of the inflammatory response in cardiovascular disease. Hypertension 2004; 43: 924 031

46. Zalba G, San José G, Moreno M, Fortuño M, Fortuño A, Beaumont F, Díez J. Oxidative stress in arterial hypertension. Role of $\mathrm{NAD}(\mathrm{P}) \mathrm{H}$ oxidase. Hypertension 2001; 38: 1395 - 1399

47. Miyamoto Y, Saito Y, Kajiyama N, Yoshimura M, Shimasaki Y, Nakayama $\mathrm{M}$ et al. Endotelial nitric oxide synthase gene is positively associated with essential hypertensio. Hypertension 1998; 32: 3-8

48. Lacy F, Kailasam MT, O'Connor DT, Schimd-Schöenbein GW, Parmer RJ. Plasma hydrogen peroxide production in human essential hypertension. Role of heredity, gender and ethnicity. Hypertension 2000; 36: $878-884$

49. Lüscher T, Barton M. Endothelins and endothelin receptor antagonists. Therapeutic considerations for a novel class of cardiovascular drugs. Circ 2000; 102: 2434 - 2440

50. Tiret L, Poirier O, Hallet V, et al. The Lys198Asn polymorphism in the endothelin-1 gene is associated with blood pressure in overweight people. Hypertension 1999; 33: 1169 - 74

51. Schiffrin E. Role of endothelin-1 in hypertension. Hypertension 1999;34:876-881.

52. Elijovich F, Laffer CL, Gavras H, Bresnahan MR, Schiffrin EL. Regulation of plasma endothelin by SALT in salt-sensitive hypertension. Hypertension 1999; 33: 1075. Abstract 